\title{
Occupational Therapy Association of South Africa Position Statement on Rehabilitation
}

\section{INTRODUCTION AND PURPOSE}

The World Health Organization positions rehabilitation as "instrumental in enabling people with limitations in functioning to remain in or return to their home or community, live independently, and participate in education, the labour market and civic life ${ }^{\prime \prime}$.

The purpose of this paper is to state the Occupational Therapy Association of Occupational Therapy's (OTASA) position on rehabilitation and how rehabilitation relates to the profession's scope of practice.

\section{STATEMENT OF POSITION}

I. The term rehabilitation is understood to include habilitation (referring to people who acquire disabilities congenitally or early in life).

2. There is convincing evidence that early, intensive and coordinated multi-disciplinary rehabilitation significantly reduces the impact of a broad range of health conditions on functioning and thus disability.

OTASA regards rehabilitation as the essential link between medical intervention and the resumption of participation in a full round of daily occupations which in turn brings meaning, purpose and satisfaction in life and allows the individual to realise his / her potential.

3. OTASA regards access to rehabilitation as a human rights issue. This position is informed by the Constitution of the Republic of South Africa ${ }^{2}$, the United Nations Convention on the Rights of Persons with Disabilities ${ }^{3}$ as well as the core values ${ }^{a}$ of the profession.

All people have the right to access appropriate health care. In addition, viewed from an occupational therapy perspective, all people have the right to:

- Participate in a range of occupations $s^{b}$ that enable them to flourish, fulfil their potential, and experience satisfaction in a way consistent with their culture and beliefs ${ }^{4}$.

- Engage fully in society.

In its essence, the outcome of rehabilitation is resumption of a full and meaningful life and preservation of the dignity of the individual.

4. OTASA affirms that:

- Rehabilitation is required when a health condition or barriers in the environment result in residual impairment, activity limitations or participation restrictions.

- People across the age spectrum with a variety of health conditions (physical / mental health / sensory / developmental) may require rehabilitation.

- Rehabilitation typically commences once the health condition is stabilised. It is a process which may have multiple

${ }^{a}$ Altruism, equality, freedom, justice, dignity, truth and prudence are generally accepted as the core values of occupational therapy.

bIn occupational therapy, occupation refers to the everyday activities that people do to occupy their time and bring purpose and meaning to life. Occupations include things that people need to, want to, or are expected to do. stages and culminates with integration into family, community and economic life.

- Early access to rehabilitation is required to achieve the best possible outcome.

- The knowledge, skills and expertise of a variety of categories of health care and other professionals may be required, simultaneously or at different times, during rehabilitation.

- Rehabilitation reaches beyond the health sector, encompassing and including the involvement of many other sectors e.g. education, transportation, social services, labour, housing.

- Rehabilitation occurs in a variety of settings - hospitals, specialised rehabilitation units, in the community, in homes, schools and work places.

- Rehabilitation is a goal-oriented and time-limited process that unfolds over a few focussed intervention sessions or gradually over a longer period of time with intermittent breaks between intervention sessions.

\section{OCCUPATIONAL THERAPY AND REHABILITATION}

I. Occupational therapists are committed to advance the right of all people - including people with disabilities - to develop their capacity and power to construct their own destiny through occupation.

Occupational therapists have particular expertise and a set of skills which are used to:

- Explore the individual's pre-morbid occupational profile.

- Address limitations in functioning which impact on ability to perform daily occupations.

- Identify challenges / obstacles to occupational engagement and find solutions to these challenges / obstacles.

Strategies may include (but are not restricted to):

- Changing the manner in which occupations are performed.

- Modifying the environment.

- Using assistive technology.

- Facilitate engagement in a full round of necessary and preferred daily occupations.

The broad goals of rehabilitation and occupational therapy are very closely aligned.

2. Consistent with occupational therapy's commitment to clientcentred intervention, the individual receiving rehabilitative intervention is centrally placed in the rehabilitation team. Rehabilitation cannot be imposed. The individual is viewed as a respected partner and his / her involvement and selfdetermination regarding all aspects of the rehabilitation process is imperative.

The involvement of family members / caregivers is also important.

3. In addition to facility-based rehabilitation programmes, occupational therapists are committed to community based rehabilitation (CBR) - a strategy within community development for the rehabilitation, equalisation of opportunities and social integration of people with disabilities ${ }^{5}$. 
Occupational therapists may be involved in CBR in training / education or working 'hands-on' in communities to promote disability inclusive development and social inclusion through occupation-based strategies.

4. Occupational therapists have a particular role to play in vocational rehabilitation - a rehabilitation strategy that aims to "enable a disabled person to secure, retain and advance in suitable employment and thereby to further such person's integration or reintegration into society" 6 .

Services provided in vocational rehabilitation depend on the needs and goals of the client, employer or insurer, and the environment in which the work takes place.

5. Occupational therapists contribute to psychosocial rehabilitation - a recovery orientated process that helps individuals who have a mental health condition that seriously impairs their ability to lead meaningful lives to gradually develop life skills, and access the resources needed for social inclusion. Psychosocial rehabilitation assists persons with psychiatric disability to increase their capacity to be successful and satisfied in the living, working, learning, and social environments of their choice $^{7}$. Occupational therapists promote mental health and recovery by facilitating goodness of fit between the person, the environment and the occupations that he or she wants to and needs to engage in.

6. Occupational therapists have a role to play in educational rehabilitation and habilitation. They promote inclusive education $^{8}$ for children and youth with disabilities or special learning needs by providing developmental, preventative and remedial interventions that foster development through engagement in the occupations of childhood including play and learning.

\section{REFERENCES}

I. World Health Organization. Rehabilitation. Disability and Rehabilitation; 2016. http://www.who.int/disabilities/care/en/ (2 Nov 2016).

2. Republic of South Africa. The Constitution of the Republic of South Africa No. 108; 1996. http://www.justice.gov.za/legislation/constitution/saconstitution-web-eng.pdf (2 Nov 2016).

3. United Nations. Convention on the Rights of Persons with Disabilities. New York: United Nations; 2006.

4. World Federation of Occupational Therapists. Position Statement on Human Rights. World Federation of Occupational Therapists; 2006.

5. World Federation of Occupational Therapists. Position Statement on Community-based Rehabilitation. World Federation of Occupational Therapists; 2004

6. International Labour Organization. CI59 - Vocational Rehabilitation and Employment (Disabled Persons) Convention (No. 159); 1983. http://www.ilo.org/public/english/standards/relm/ilc/ilc86/r-iii lba. htm (2 Nov 2016).

7. Anthony WA, Farkas MD. A Primer on the Psychiatric Rehabilitation Process. Boston, MA: Center for Psychiatric Rehabilitation, Boston University; 2009.

8. Department of Education. Education White paper 6 Special needs education: Building an inclusive education and training system. Pretoria: Department of Education; 200I. https://wcedonline. westerncape.gov.za/Specialised-ed/documents/WP6.pdf (27 Mar 2017).

DATE RATIFIED

13/07/2016; Revised 22/04/2017 